\title{
Cognitive Radio: An Emerging trend for better Spectrum Utilization
}

\author{
Atul Singh Jamwal \\ Deptt. Of Computer Science \\ SVIET, Banur \\ India
}

\author{
Gurpinder Kaur \\ Deptt. Of Computer Science \\ SVIET, Banur \\ India
}

\begin{abstract}
Due to the rapid development of wireless communications in recent years, the demand on wireless spectrum has been growing dramatically, resulting in the spectrum scarcity problem. Works have shown that the fixed spectrum allocation policy commonly adopted today suffer from the low spectrum utilization problem. Both academic and regulatory bodies have focused on dynamic spectrum access to fully utilize the scarce spectrum resource. Cognitive radio, with the capability to flexibly adapt its parameters, has been proposed as the enabling technology for unlicensed secondary users to dynamically access the licensed spectrum owned by legacy primary users on a negotiated or an opportunistic basis. In this paper we present a volumetric survey on various methods used to adapt changes used in cognitive radio.
\end{abstract}

Key words: Cognitive Radio, spectrum holes, primary users, spectrum sensing, network management.

\section{INTRODUCTION}

Cognitive radio network is a new emerging research area recently. It enhances the existing software-defined radio, whose physical layer behavior is largely defined in software. Cognitive radio has the following characteristics. [3] First, it is aware of its environment and its capabilities. Second, it is able to independently alter its physical layer behavior based on its previous experience and its current environment. Finally, it is capable of performing the complex adaptation strategies according to the cognitive cycle shown in. With these capabilities, when spectrum environment changes around cognitive user, it is capable of sensing these changes and independently changing its physical layer settings such as transmission power, channel selection and etc to meet some constraints or requirements of the users.

Cognitive radio gains popularity in the research area because it enables [4] the current fixed spectrum channel assigned by FCC to be utilized by the new users. For example, most of the spectrums assigned to TV channels are idle most of the time, while wireless network users share a small range of spectrum, 2.4 G Hz and 5G Hz. When there are many wireless users at a time, the network is congested because of the limited channel. With the spectrum opportunities provided by the cognitive radio network, the wireless network users are able to share the idle spectrum for TV channel, on the condition that it does not interfere with the normal TV channel.

\section{BACKGROUND}

The concept of cognitive radio was first proposed by Joseph Mitola III [1] in a seminar at KTH (the Royal Institute of Technology in Stockholm) in 1998 and published in an article by Mitola and Gerald Q. Maguire, Jr. in 1999. It was a novel approach in wireless communications, which Mitola later described as: "The point in which wireless personal digital assistants (PDAs) and the related networks are sufficiently computationally intelligent about radio resources and related computer-to-computer communications to detect user communications needs as a function of use context, and to provide radio resources and wireless services most appropriate to those needs". In November 2002, Federal Communication Commission (FCC) of USA first prepared a report which lists some of the provisions for spectrum management in Cognitive Radio [2]. In this report FCC described some regulatory policies for the efficient use of Spectrum under Cognitive Radio. FCC noted that utilization of radio network is not balanced. Some frequency bands are overloaded and some are under loaded, creating inefficient spectrum utilization. Now the task was to make the network intelligent enough to adapt dynamically in overloaded or under loaded conditions without the interference of users.

\section{ISSUES IN COGNITIVE RADIO}

\subsection{Cognitive Radio and Spectrum Sensing}

The most important aspect of using cognitive radio is the ability to sense the unused spectrum. Spectrum Sensing can be 
defined as the [4] combination of signal detection and modulation classification and use the general term automatic modulation classification (AMC) to denote this combined process.

Spectrum sensing can also be defined on the basis of interference temperature. An unused spectrum has different sensing temperature as compare to used one [1]. This difference is due to interference and has different frequency bands. An unused spectrum can be sensed using this frequency band difference.

Spectrum holes are another ways of finding the unused spectrum. Spectrum holes are created where an unused band is present. Identify these spectrum holes can make the spectrum easily sensed for unused bandwidth. All channels are classified into different spectrum holes. These are:

- White spectrum holes, which are not fully used.

- Grey spectrum holes which are partially used.

- Black spectrum holes which are fully used.

After detecting the channel unused, a user can freely use the white spectrum holes and partially used the grey spectrum holes and black spectrum holes are not used at all as it is assumed that these types of holes are fully used by primary or licensed users. The use these fully used spectrum holes may hampers the communication of primary users.

\subsection{Cognitive Radio and Spectrum Decision}

When the unused channel is found the next step will be the spectrum decision by which cognitive radio must find the initial point of its operation and select the appropriate technical parameters according to the present state of the environment in which it has to operate on. This spectrum decision is very crucial as if wrongly made can interrupt the communication of primary users. Cognitive radio is assumed to have the capability to self adjust dynamically according to its changing environment characteristics and is able to freely make decision depending upon the parameters of the current environment. It means that it must have such a decision making power that come into use while operating in the changing environment. To operate with self decision making capabilities, cognitive radio must be entrenched with some kind of artificial intelligence techniques so that can take its decisions efficiently and independently without interfering the other users.

\subsection{Cognitive Radio and Spectrum Sharing}

Sharing of unused spectrum among various users is another issue while using the cognitive radio. Since there is very limited unlicensed band available and can be used by anybody, these bands are mostly overloaded and heavily used ones, because it much easier and cheaper for users to access these bands. It means decision must be chosen so not to interfere with primary users and also do not overlap with other secondary users. Sharing should be done on first come first serve manner or apply some other allocation techniques on network sharing on priority basis to increase the spectrum utilization. Moreover spectrum sharing can be done by giving each secondary user a time quantum within which he must have to finish his network use and handover the network to be used by other users. Sharing of unused bandwidth with proper coordination and follow some regulatory rules and other policies should be done in efficient and altruistic manner.

\subsection{Cognitive Radio and Network Security}

One of the major issues concerned with cognitive radio is network's vulnerability to be open to all the users whether they are authorized or unauthorized. Since network is freely accessible to malicious users they can interrupt the primary authorized users by interfering between their spectrums. A cognitive radio should also have some security mechanisms to recognize the users of the network. A cognitive radio network should also be imparted with encryption techniques to communicate securely. To address these security issues regulatory bodies must have some provisions or policies to use the spectrum safely and securely.

\subsection{Spectrum Management}

The key challenge in implementing a cognitive radio network is allocation of frequency bands to different users. Licensed users generally have stable frequency band whereas unlicensed users use dynamically allocated bands. It is quite difficult to distribute unused frequency bands which reside between stable bands as it may interrupt the primary users. There must be some heuristic algorithms to allocate a sequence of dynamically changing band which also keep these bands away from stable bands. 


\section{CONCLUSION}

Although cognitive radio is an emerging trend for utilizing the spectrum efficiently still there are some concerns which must be addressed to use it best possible. It would be recommended if cognitive radio is used with some powerful network security techniques so that its use does not interrupt the primary users. Moreover some strict provisions must be implemented by the regulatory bodies for effective and efficient use of this technique.

\section{REFERENCES}

[1]. Joseph Mitola et al "Cognitive Radio: Making Software Radios More Personal” IEEE 1999.

[2]. Amna Saad Kamil,Ibrahim Khider "Open Research Issues in Cognitive Radio" $16^{\text {th }}$ Telecommunication Forum TELFOR 2008.

[3]. Simon Haykin "Cognitive Radio: Brain-Empowered Wireless Communications" IEEE Journal on selected areas of communication Feb 2005.

[4]. Allen B. MacKenzie et al "CognitiveRadio and Networking Research at Virginia Tech” IEEE 2009.

[5]. Simon Haykin "Cognitive Radio: Research Challenges" VTC sep 2008.

[6]. T. Charles Clancy "Software Defined Radio and Cognitive Radio” MILCOM 2007.

[7]. Beibei Wang, Yongle Wu, K.J. Ray Liu "Game theory for cognitive radio networks: An overview" ELSEVIER april 2010.

[8]. William Krenik and Anuj Batra "Cognitive Radio Techniques for Wide Area Networks” DAC 2005.

[9]. Jayakrishnan Unnikrishnan "Cooperative Sensing for Primary Detection in Cognitive Radio” IEEE 2008.

[10]. Ian F. Akyildiz et al "A Survey on Spectrum Management in Cognitive Radio Networks" IEEE Communications Magazine April 2008.
[11]. Xiao Yu Wang et al, "Extended Knowledge-Based Reasoning Approach to Spectrum Sensing for Cognitive Radio” IEEE Transactions on Mobile Computing April 2010. 